\title{
Cyclostrophic vortices in polar regions of rotating planets
}

\author{
V. Goncharov ${ }^{1}$ and V. Pavlov ${ }^{2}$ \\ ${ }^{1}$ Institute of Atmospheric Physics, Russian Academy of Sciences, 109017 Moscow, Russia \\ ${ }^{2}$ UFR de Mathématiques Pures et Appliquées, Université de Lille 1, 59655 Villeneuve d'Ascq, France
}

Received: 10 January 2001 - Revised: 17 April 2001 - Accepted: 20 April 2001

\begin{abstract}
Multi-petal, rotating vortices can form in twodimensional flows consisting of an inviscid incompressible fluid under certain conditions. Such vortices are principally nonlinear thermo-hydrodynamical structures. The proper rotation of these structures which leads to time-dependent variations of the associated temperature field can be enregistred by a stationary observer. The problem is analyzed in the framework of the contour dynamics method (CDM). An analytical solution of the reduced equation for a contour curvature is found. We give a classification of the solutions and compare the obtained results with observational data.
\end{abstract}

\section{Introduction}

In this paper, we elucidate conditions under which largescale, stationary rotating structures which consist of "hot" vortex formations of the multi-petal type can form. Such vortices are principally nonlinear thermo-hydrodynamical structures. The proper rotation of these structures which leads to time-dependent variations of the associated temperature field can be enregistred by a stationary observer.

Large-scale, long-lived vortices are found in many types of hydrodynamic flows. The problem of the formation and of the evolution of such vortives in turbulence, known at coherent structures, attracts much attention. Large-scale vortices are largely observed in the planetary atmosperes and in the oceans (see Gill, 1982; Robinson, 1983; Ingersoll, 1990, and references therein). On the one hand, we see evidence of self-organisation in nature. On the other hand, large-scale oceanic and atmospheric vortices attract interest due to the role they can play, par example, in the transport of heat, salt and other properties.

A question immediatelly arises: under what conditions do large-scale vortex structures form? In traditional statistical approachs, the turbulent medium is usually considered to be homogeneous and isotropic. Common sense and thermody-

Correspondence to: V. Pavlov (vadim.Pavlov@uni-lille1.fr) namical laws suggests that is very difficult to extract energy from a chaotic system, and only with some additional specific properties of such systems is this possible to archive. Homogeneous, isotropic turbulence, which does not possess any distinguishing scales or preferred directions, is too symmetric to give birth to large-scale vortices; self-organization seems to be improbable in this case. It is evident that the breaking of some symmetry is a necessary condition for selforganisation. It is clear, par example, that turbulent fluid with broken spherical symmetry (quasi two-dimensional approximation) can be considered a suitable candidate of a medium where large-scale vortices can be organized. In line with this remark, we consider only hereinafter the quasi twodimensional approximation: the flows which commonly occur in nature where the horizontal velocity components dominate over the vertical velocity.

In the geophysical context, there are several possible reasons for the depletation of one (vertical) of the velocity components of the flow: the two-dimensional motion is cuased by the pre-dominant two-dimensional nature of the flow domain itself, when tbe characteristic horizontal scale of the flow, $L$, greatly surpasses the vertical one, $H_{s}$ (the so-called thin-layer atmospheric models when $\epsilon \equiv H_{S} / L \ll 1$ ). The depletation can also be due to the strong density stratification, or due to the rotation of the system as a whole, shallow layers. In electrically conducting fluid (plasma flows in magnetic fields), such a depletation is ensured by a strong external magnetic field. The depletation of one velosity component is also observed in experimental systems, including thin layers of electrolytes, and flows in soap films.

In the atmosphere and oceans of the Earth, the quasi twodimensional large-scale vortex structures are abundant. Examples of such structures are the Golf Stream rings, the vortices shed from coastal currents, the cyclones and anticyclones, the Antarctic Polar Vortex, etc. Two-dimensional models $\epsilon \equiv H_{S} / L \rightarrow 0$ are often applied to such systems (Kamenkovich, 1977; Pedlosky, 1987, and references therein).

For atmospheres, the following characteristic scales of 
the field variables based on observed values can be introduced (Holton, 1992): the horizontal velocity scale $\langle v\rangle \sim$ $1 \div 10 \mathrm{~m} \mathrm{~s}^{-1}$, the vertical velocity scale $\langle w\rangle \sim 1 \mathrm{~cm} \mathrm{~s}^{-1}$, the length scale $L \sim 10^{5}-10^{6} \mathrm{~m} \ll R_{0} \sim 10^{7} \mathrm{~m}$, and the height scale $H_{S} \sim 10^{4} \mathrm{~m}$. Since the vertical velocity component $\langle w\rangle$ is small, $|w| \sim \epsilon$, where $\epsilon=H_{s} / L \ll 1$, we are limited by the lowest approximation when all small terms $\sim \epsilon$ are omitted in the governing equations of motion. Thus, the evolution of larger scales, where $L \gg H_{s}\left(L \ll R_{0}\right)$ for large vortex structures in the atmosphere can be considered two-dimensional in the lowest approximation.

On the other hand, the approximation of a quasi-ideal fluid is justified in real flows, including turbulent ones, if the characteristic scales of the vortex structures are greater than the characteristic scales of turbulent pulsations (Pedlosky, 1987). The atmospheric large-scale flows are characterized by ultralarge Reynolds numbers $R e=v L / v \gg 1$. If $L \sim 10^{5} \mathrm{~m}$, and $v \sim 1 \mathrm{~m} / \mathrm{s}$, even if the kinematic viscosity $v$ is replaced by a turbulent viscosity, one has $R e \sim 10^{6} \div 10^{8} \gg 1$. Such flows have the striking property of organizing spontaneously into large-scale coherent vortex structures. A well-known example is Jupiter's Great Red Spot, a huge vortex with a very large Reynolds number and intense small-scale turbulent motion, which has persisted for more three centuries; the presence of intense turbulence does not destroy it. Let us note that even if a flow is turbulently unsteady (due to convection, buoyancy, etc.), this does not necessarily mean that the average ordered motion is absent. In such a case, the equations of motion of the ideal (see below), or quasi-ideal fluid with "eddy" viscosity are known to be a good approximation of the description of large-scale average fields of a turbulent flow.

Two-dimensional Euler flows $(R e \rightarrow \infty)$ are the inviscid limit of two-dimensional incompressible Navier-Stokes flows. They are often used as the simplest models for geophysical and astrophysical flows (Dritschel and Legras, 1993; Frisch, 1995; Marcus, 1988; Sommeria et al., 1988; Pavlov et al., 2001; Goncharov and Pavlov, 2001).

The assumption that all field variables are independent of the coordinate $z \equiv x_{3}$, i.e. the operator $\partial_{3} \equiv 0$ for all field variables, also leads to two-dimensional models and is largely used in theoretical considerations (Milne-Thomson, 1968; Greenspan, 1968; Lavrent'ev and Shabat, 1973).

We start with a reminder of some observational data. For Earth and Venus and their atmospheres, some basic properties are: the rotation periods $2 \pi / \Omega$ are $23.9 \mathrm{hr}$ (prograde) and 243 days (retrograde); the overhead motions of the Sun are east to west for the Earth and west to east for Venus (inclinations of equator to orbit are $\sim 23^{\circ}$ and $\sim 177^{\circ}$ ); the radii $R_{0}(\mathrm{~km})$ are $\sim 6360$ and $\sim 6052$; the gravity accelerations $g$ $\left(\mathrm{m} \mathrm{s}^{-2}\right)$ are $\sim 9.8$ and 8.9 (at surface); the surface temperatures $T_{S}(\mathrm{~K})$ are $\sim 288$ and $\sim 730$; the surface pressures $p_{s}$ (Mpa) are 0.1 and 9.2; the densities near the surface $\rho_{s}\left(\mathrm{~kg} \mathrm{~m}^{-3}\right)$ are 1.23 and 65.0; the pressure scale heights $H_{S}(\mathrm{~km})$ (at surface) are $\sim 8.4$ and $\sim 15.8$.

Observational data provides the evidence (Seiff, 1983) that the lower atmosphere of Venus $(\leq 70 \mathrm{~km})$ is generally statically stable except for limited altitude intervals. Except for two intervals, the data displays an atmosphere which is stable rather than convective, whereas prior to the measurements, a convective atmosphere had been generally expected.

Due to Venus's very slow rotation, the winds in its atmosphere are subjected to a weak Coriolis force. The relative importance or unimportance of the Coriolis force in the atmospheric motions can be characterized quantitatively by evaluating the Rossby number $R o=V / L f$, where $V$ is the horizontal wind speed, $L$ is the horizontal length scale of the fluid motion, and $f=2 \Omega \sin \theta$ is the Coriolis parameter. Here, $\Omega$ is the planetary rotation rate and $\theta$ is the latitude. The Rossby number is the ratio of the inertial force to the Coriolis force. If $R o \ll 1$, the motion is quasigeostrophic and the Coriolis force exerts a strong control on the atmospheric motions (on rapidly rotating planets, such as the Earth or Jupiter); if $R o \gg 1$, the Coriolis force is negligible. Typical values of $V$ and $L$ for large-scale motions can be proposed as $10 \mathrm{~m} \mathrm{~s}^{-1}$ and $10^{5}-10^{6} \mathrm{~m}$. With $L \sim 600 \mathrm{~km}, \theta \sim 45^{\circ}, \Omega_{V} \sim 3 \cdot 10^{-7}$ and $\Omega_{T} \sim 7.3 \cdot 10^{-5}$, we find $R o \sim 30$ and $\sim 0.1$ for Venus and Earth, respectively.

In particular, it follows that for the steady regime, $\partial_{t} \boldsymbol{v}_{s}=$ 0 , the Venus atmosphere at middle latitudes cannot be in a quasi-geostrophic dynamical equilibrium when the horizontal pressure gradient is balanced by the horizontal component of the Coriolis force (for the Earth's atmosphere, see, for example, Pedlosky, 1987; Holton, 1992). The hypothesis of a cyclostrophic equilibrium is more reasonable in such a situation, where the horizontal pressure gradient is balanced by the centrifugal force (Schubert, 1983). However, it appears that the cyclostrophic balance of the zonal winds is not consistent with the observational data at latitudes near the poles.

It has been discovered that after the flights of the PioneerVenus probes (see Hunten et al., 1983; Taylor et al., 1980; Gryanik, 1990, and references quoted therein) in polar regions of Venus, the circulation and the temperature distribution have some distinguishing features. The large polar temperature contrasts which are dynamically significant suggests a more complex fluid dynamics.

Radio and infrarared data (Hunten et al., 1983) indicate a polar atmosphere appreciably colder than that at low latitudes by $\sim 20 \div 40 \mathrm{~K}$. These data show essentially no change in the average temperature $\langle T\rangle$ with latitudes up to $55^{\circ}$, and a nearly constant rate of colder $(d\langle T\rangle / d \theta)_{p}$ of $0.9 \mathrm{~K}$ per degree of latitude at higher latitudes (Hunten et al., 1983).

Data indicate a temperature minimum at the altitude level corresponding to the $200 \mathrm{mbar}$ level at a $75^{\circ}$ latitude, which is $35 \mathrm{~K}$ colder than the quatorial region at this pressure level, and $15 \mathrm{~K}$ colder than the $60^{\circ}$ latitude. The latitude of the temperature minimum corresponds closely to the central latitude of the brightest emission from the twin polar height-emission spots (Taylor et al., 1980). These two "hot spots" (of about $5^{\circ}$ in radius, i.e. $L \sim 10^{2} \div 10^{3} \mathrm{~km} \gg H_{s} \sim 10 \mathrm{~km}$ ) are positioned symmetrically with respect to the pole (at latitudes of $\sim 75-85^{\circ}$ ) and are rotated around the pole. The sym- 
metry of the temperature contours suggests that the twin hot spots may be a pair of vortices.

One of the physical explanations of the phenomenon may be as follows. Disturbances with a horizontal scale $L$ such that $L \ll R_{0}$ and $L \gg H_{s}$, which contain hotter and therefore lighter gas, have to move in the centrifugal-force field toward the axis of rotation, i.e. toward the pole. The accumulation of hot gas at the pole leads to the formation of a "hot cap". However, if localized disturbances of the fluid (hotter than the surrounding medium), positioned symmetrically with respect to pole, are vortical in nature, then their displacement directly towards the pole is obviously impossible. In fact, in the vicinity of the pole, each eddy is subjected to an influence of a velocity field induced by all other vortices. The eddies participate, therefore, in the mutual rotation about the pole in connection with their temperature anomalies. The rotation of the two- or multi-petal "hot polar cap" which is formed over the pole leads to variations in temperature that may be measured by a stationary observer. Motivated by these observations, we consider the "over-all picture" of the phenomenon.

We consider below quasi two-dimensional flows in a thin sheet of an ideal fluid, an assumption which allows one to make some additional simplifications (see Goncharov and Pavlov, 1997, 1998, 2000, and references therein).

We replace the real continuous velocity profile by a patchlike model with constant vorticity in each patch (see Zabusky et al., 1979; Saffman, 1992, and references therein). Such a model is valid when large-scale motions are weakly sensitive to a fine structure in the velocity field. In this scenario, one may expect that even crude approximations with a small number of patches, that only describe the general structure of the real vorticity profile, correctly grasp large-scale dynamics. The proposed model allows one to formulate the dynamics of the flow in terms of a small number of interfaces, while determining the contours of the vortex structures within the framework of spatially one-dimensional integro-differential nonlinear equations. This approach is called the contour dynamics method. The equations of the contour dynamics describe the self-induced motion of the vorticity-discontinuity boundaries, or "contours", in an inviscid, incompressible, two-dimensional fluid with piecewise constant vorticity distribution.

Recall that detailed comparisons between results obtained within the framework of the contour dynamics and conventional numerical simulations (see, for example, Zabusky et al., 1979; Dritschel, 1988; Dritschel and McIntyre, 1990; Saffman, 1992, and Refs therein) have shown a surprisingly good agreement for flows with distributed vorticity at ultralarge Reynolds number, $\ln R e \gg 1$. Thus, it appears that many general aspects of the nearly inviscid flows with distributed vorticities can be reproduced using contour dynamics with a moderate number of vorticity levels. These results suggest that contour dynamics may be a competitive analytical tool for problems of scientific interest, particularly in connection with large-scale, and very large Reynolds number flows.
The limits of validity for our model are defined by a formulated goal: elucidate qualitative conditions under which a large-scale, stationary rotating structure can be formed consisting of "hot" vortex formations of the multi-petal type. Instead of massaging the computer-calculated details of the vortex structures, it is more important to answer the practical question of whether the vortex may form under certain conditions. The description is tailored to obtain the basic characteristics of the vortex patch-like structures, such as the horizontal space-scale, the period of rotation of the multi-petal "hot vortex spots", etc., and even in a simplified situation, we can find a rich pattern of vortex dynamics.

A brief outline of the paper is as follows. Section 2 is devoted to the basic elements of vortex dynamics in the presence of centrifugal forces. The governing equations for steadily rotating vortex structures are presented in Sect. 3. In Sect. 4, we outline the key points of the general approach for the application of the contour dynamics method to the two-dimensional models of fluid dynamics. The method is applied to localized vortex structures in Sect. 5, where the governing equations for contour curvature are obtained. Section 6 classifies the resulting solutions, called $N$-petal vortex structures. In Sect. 7, we discuss some features of the temperature distribution associated with the multi-petal vortex patches.

\section{Basic equations and concepts}

The equations of motion for a continuum inviscid fluid express the conservation of mass, of momentum, and of energy (see, for example, Landau and Lifshitz, 1987). We express these equations in a rotating, with the angular velocity $\boldsymbol{\Omega}=(0,0, \Omega)$, Cartesian coordinate system (with the $x \equiv x_{1}$ and $y \equiv x_{2}$ axes in the horizontal plane, and $z$ vertically upwards). For such a fluid, we have the following equations (see Greenspan, 1968)

$$
\begin{aligned}
& \rho\left[\partial_{t} v_{i}+v_{j} \partial_{j} v_{i}+2 \varepsilon_{i 3 k} \Omega v_{k}\right]=-\partial_{i} p-\rho \partial_{i} \Phi, \\
& \partial_{t} T+\left(v_{j} \partial_{j}\right) T=0, \\
& \partial_{j} v_{j}=0 .
\end{aligned}
$$

Here, $v_{i}$ are the components of a velocity field, $\rho$ is the density, $p$ is the pressure, $T$ is the temperature, $\Phi=g z-$ $(1 / 2)|[\boldsymbol{\Omega}, \boldsymbol{r}]|^{2},[\boldsymbol{a}, \boldsymbol{b}]$ is the vector product of the two vectors $\boldsymbol{a}$ and $\boldsymbol{b}, \varepsilon_{i 3 k}$ is the alternating tensor (zero for any two indices being equal, +1 for any even number of permutations, and -1 for any odd number). The application of the operator $\nabla \times$ to the equation of momentum leads to an equation

$$
\left[\nabla+\beta \nabla T, \frac{d \boldsymbol{v}}{d t}+2[\boldsymbol{\Omega}, \boldsymbol{v}]\right]=\beta\left[\nabla\left(\tau_{s}+\tau\right), \nabla \Phi\right] .
$$

Here, $\boldsymbol{r}=\left(\boldsymbol{x}, x_{3}\right), v_{j}=(\boldsymbol{v}, w), \quad \nabla \times \boldsymbol{s} \equiv[\nabla, \boldsymbol{s}], \quad \nabla \times$ $(\rho \boldsymbol{s})=\rho \nabla \times \boldsymbol{s}+[\nabla \rho, \boldsymbol{s}], \Phi=g z-(1 / 2)|[\boldsymbol{\Omega}, \boldsymbol{r}]|^{2},[\boldsymbol{a}, \boldsymbol{b}]$ is the vector product of the two vectors $\boldsymbol{a}$ and $\boldsymbol{b}, \nabla \rho=$ $(\partial \rho / \partial T)_{p} \nabla T+(\partial \rho / \partial p)_{T} \nabla p \simeq-\rho \beta \nabla T$. The coefficient 
of thermal expansion, $\beta=-\rho^{-1}(\partial \rho / \partial p)_{T}$, is assumed constant. The rotation axis of the fluid coincides with the $z$-axis, the vector $\boldsymbol{g}$ is anti-parallel to the vertical axis, and is perpendicular to the plane $x 0 y$. The departures of the pressure $p^{\prime}=p-p_{0}$ and density $\rho^{\prime}=\rho-\rho_{0}$ from their undisturbed values $p_{0}$ and $\rho_{0}$ are assumed small enough. For the equation of state we shall assume, in accordance with the assumption of an incompressible medium, that the density depends only on the temperature and not on the pressure. Thus, we set $\rho=\rho_{0}\left[1-\beta\left(T-T_{0}\right)\right]$, where subscript 0 denotes the reference values. Because $\beta\left(T-T_{0}\right)$ is generally of little interest here, one may neglect the density variations and hence replace $\rho$ by the constant value $\rho_{0}$, except in the "buoyancy" term $\sim \partial_{i} \Phi$ (see, for example, Turner, 1979; Landau and Lifshitz, 1987).

Let be $\epsilon_{1}=\beta|\Delta T| \ll 1$. In this case, the term $\beta[\nabla T, s]$ is small with respect to $[\nabla, s]$ on horizontal scales and can be neglected. Limiting by this approximation, we obtain the equation

$\left[\nabla, \frac{d \boldsymbol{v}}{d t}+2[\boldsymbol{\Omega}, \boldsymbol{v}]\right]=\beta\left[\nabla\left(\tau_{s}+\tau\right), \nabla \Phi\right]$.

In the tensorial form, this equation can be rewritten as

$\mathcal{D}_{t} \omega_{i}=\omega_{j} \partial_{j} v_{i}+\beta \varepsilon_{i j k} \partial_{j}\left(\tau_{s}+\tau\right) \partial_{k} \Phi$.

Here, $\mathcal{D}_{t}=\partial_{t}+v_{j} \partial_{j} \equiv D_{t}+w \partial_{3}, \quad j=1,2,3, \quad D_{t}=$ $\partial_{t}+(\boldsymbol{v} \cdot \nabla), \boldsymbol{r}=\left(\boldsymbol{x}, x_{3}\right), v_{j}=(\boldsymbol{v}, w), \omega_{i}=\varepsilon_{i j k} \partial_{j} v_{k}+$ $2 \Omega_{i}, \quad \tau_{s}+\tau=T-T_{0}, \quad \tau_{s}$ is a part of the temperature perturbation independent of the time.

Noting that $\varepsilon_{3 j k} \partial_{j} \tau_{s} \partial_{k} \Phi \equiv 0$ because of a postulated axial symmetry of $\tau_{s}$, we obtain for $\omega_{3}$ and $\tau$ the basic set of equations:

$D_{t} \omega_{3}=\beta \varepsilon_{3 j k} \partial_{j} \tau \partial_{k} \Phi$,

$D_{t} \tau=-(\boldsymbol{v} \cdot \nabla) \tau_{s}$,

$\nabla \cdot v=0$.

Here, $D_{t}=\partial_{t}+(\boldsymbol{v} \cdot \nabla), \boldsymbol{v}=\left(v_{1}, v_{2}\right), \boldsymbol{\nabla}=\left(\partial_{1}, \partial_{2}\right)$ is the horizontal gradient operator, $\omega_{3}=\varepsilon_{3 j k} \partial_{j} v_{k}+2 \Omega_{3}, \tau_{s}+$ $\tau=T-T_{0}, \tau_{s}$ is an axially symmetrical part of the temperature perturbation which is independent of the time.

We consider the simplest model in which the vortex structures are formulated in a "thin" flat sheet of an incompressible inviscid fluid when the vertical component $w$ of the velocity is depleted and can be neglected (i.e. $H_{s} \ll L, V^{2}$ $\ll g L, \operatorname{Re} \gg 1$; see, for example, Kamenkovich, 1977; Pedlosky, 1987; Goncharov and Pavlov, 1997).

The condition of incompressibility (1) makes it possible to express velocity components in terms of the stream function $\psi$ :

$v_{i}=\varepsilon_{i k} \partial_{k} \psi$

Here, $i=1,2, \varepsilon_{i k} \equiv-\varepsilon_{i 3 k}$ is the antisymmetric unit tensor of the second order, $\varepsilon_{12}=-\varepsilon_{21}=1, \varepsilon_{11}=\varepsilon_{22}=0$. In this case, we have $\omega_{3}=-\Delta \psi$, where $\Delta=\partial_{1}^{2}+\partial_{2}^{2}$. To simulate the effect of the temperature stratification, i.e. the increasing of the stationary temperature with respect to distance from axe of rotation, we take the background distribution of the temperature $\tau_{s}$ in the form (here $r=|\boldsymbol{x}|, \boldsymbol{x}=\left(x_{1}, x_{2}\right)$ )

$\tau_{s}(\boldsymbol{x})=\frac{\gamma}{2} r^{2}, \quad \gamma>0$.

This expression can be considered the result of expanding a radially symmetric stationary distribution $\tau_{s}(\boldsymbol{x})$ in the Taylor series in the vicinity of the axe.

We obtain the coupled set of governing equations

$\partial_{t}[\Delta \psi-2 \Omega]+\varepsilon_{i k} \partial_{i} \psi \partial_{k}[\Delta \psi-2 \Omega]=\Omega^{2} \beta \varepsilon_{i k} x_{i} \partial_{k} \tau ;$

$\partial_{t} \tau+\varepsilon_{i k} \partial_{i} \psi \partial_{k} \tau=\gamma \varepsilon_{i k} x_{i} \partial_{k} \psi$.

In the absence of temperature stratification $(\gamma=0)$ and centrifugal force $(\Omega=0)$, Eqs. (4) become traditional equations for the vortex evolution in a two-dimensional ideal fluid.

The generalization of (1)-(4) to a more complex case can be made too, for example, for a thin layer of fluid on a sphere.

\section{Steadily rotating vortex structures}

Let us consider the solutions for Eqs. (4) which correspond to the stationary vortex structures rotating with a constant angular velocity $\omega$ around the pole.

Having in mind applications, we retain $R o \gg 1$ and consider it suitable for our purpose. In this case, $\omega_{3} \simeq-\Delta \psi$. For Venus, the characteristic Rossby's number is $R o \sim 30$.

Taking into account that the structures become immovable when shifting to a new rotating coordinate system, the derivative with respect to time is transformed as

$\partial_{t}=-\omega \varepsilon_{i k} x_{i} \partial_{k}$,

and we obtain the following system of equations:

$\varepsilon_{i k} \frac{\partial}{\partial x_{i}}\left(\psi-\omega \frac{r^{2}}{2}\right) \frac{\partial \Delta \psi}{\partial x_{k}}=\Omega^{2} \beta \varepsilon_{i k} x_{i} \frac{\partial \tau}{\partial x_{k}}$,

$\varepsilon_{i k} \frac{\partial}{\partial x_{i}}\left(\psi-\omega \frac{r^{2}}{2}\right) \frac{\partial \tau}{\partial x_{k}}=\gamma \varepsilon_{i k} x_{i} \frac{\partial \psi}{\partial x_{k}}$.

It follows from (5) that the general expression for $\psi(\tau)$ should be sought in the form

$\psi=\omega \frac{r^{2}}{2}+f\left(\tau+\gamma \frac{r^{2}}{2}\right)$,

where $f(s)$ is an arbitrary function. This result is obtained after the substitution of (6) in (5) and after differentiating $\omega r^{2} / 2+f\left(\tau+\gamma r^{2} / 2\right)$ with respect to arguments. To eliminate the functional arbitrariness in (6), we shall assume that $\psi, \tau \rightarrow 0$ at $r \rightarrow \infty$. The requirement of localization for the physical fields, $\psi$ and $\tau$, namely, $\psi, \tau \rightarrow 0$ at $r \rightarrow \infty$, leads to the result that $f(s)$ is a linear function of $s$, and to the relation

$\psi=-\frac{\omega}{\gamma} \tau$. 
Substituting (7) in (5) we find the equation

$\varepsilon_{i k} \frac{\partial}{\partial x_{i}}\left(\psi-\omega \frac{r^{2}}{2}\right) \frac{\partial}{\partial x_{k}}\left(\Delta \psi-\frac{\Omega^{2} \gamma \beta}{\omega^{2}} \psi\right)=0$.

\section{Contour dynamics method}

The contour dynamics method (CDM) used in this paper is an analytical method for simulating large-scale vortex structures (regions of rotating fluid) in two-dimensional flows. CDM is based on the observation that for an inviscid, incompressible fluid, the evolution of a patch of uniform vorticity is fully described by the evolution of its bounding contour. The method is not limited to a patch of uniform vorticity: several contours can be nested in a stack. Therefore, a given continuous vorticity distribution can be approximated by a step-function. The velocity in the fluid and, in particular, at the contours of vorticity-discontinuity can be found using the Green's function of the associated Laplace (or another) operator, or be using the method of pseudo-differential operators (Maslov, 1973; Goncharov and Pavlov, 2000).

The equations of contour dynamics describe the selfinduced motion of the vorticity-discontinuity boundaries, or "contours," in an inviscid, incompressible, two-dimensional fluid whose vorticity distribution is piece-wise constant. From a mathematical viewpoint, the assumption of piecewise constant vorticity seems severe. But detailed comparisons between the results obtained both in the framework of the CDM and by direct numerical calculations show good agreement for flows with distributed vorticity with a very high Reynolds number. It appears that many aspects of the nearly inviscid flows with continuous distributed vorticity can be reproduced using contour dynamics with a moderate number of vorticity levels.

Before proceeding to analyze Eq. (8), we note that it bears a close resemblance to the well-known stationary equation for geostrophic potential vorticity in an appropriately rotating reference frame (Pedlosky, 1987). This analogy allows us to name the quantity

$q=\Delta \psi-\frac{1}{R^{2}} \psi, \quad R=\frac{|\omega|}{\Omega \sqrt{\gamma \beta}}$,

as a cyclostrophic potential vorticity and correspondingly, $R$ is the length-scale characteristic of the problem.

Let us note here that the geostrophic potential vorticity (GPV) evolves as a Lagrangian quantity with a fluid particle and hence is materially conserved. In contrast with the GPV, the quantity called the cyclostrophic potential vorticity (CPV) does not fall into this class.

Concerning the character of a spatial distribution of the quantity $q$, we shall assume that it is a piece-wise uniform function in the plane $z=x_{1}+i x_{2}$. Let us note again that $\boldsymbol{x}=\left\{x_{1}, x_{2}\right\}$. Such a distribution may be described simply by using two-dimensional Heaviside step-functions:

$\theta(z)= \begin{cases}1, & \text { if } \quad z \in D \\ 0, & \text { If } \quad z \notin D\end{cases}$
Here, $D$ is a singularly connected region in the $z$-plane bounded by a closed contour which is given in the parametric form

$z=\hat{z}(s)$,

where $s$ is contour arc length. For the vector $\hat{z}_{s}=\partial \hat{z} / \partial s$ tangential to the contour, the following normalizing condition holds

$\left|\hat{z}_{s}\right|^{2}=1$.

Note that the $\theta$-functions admit the following analytical representation through the contour integral:

$\theta(z)=\frac{i}{2 \pi} \oint d s \frac{\hat{z}_{s}}{z-\hat{z}}$.

This representation can be obtained as a corollary of the formula known in the theory of function of complex variables as Cauchy's formula. Using another formula (see Madelung, 1957):

\section{$\frac{\partial}{\partial \bar{z}} \frac{1}{z}=\pi \delta(\boldsymbol{x})$,}

the $z$-derivative of the $\theta$-function can be easily calculated from (12) as

$\frac{\partial \theta}{\partial \bar{z}}=\frac{i}{2} \oint d s \hat{z}_{s} \delta(\boldsymbol{x}-\hat{\boldsymbol{x}})$.

Thus, in terms of the $\theta$-functions, the distribution of $q$ can be written as

$q=q_{0} \theta(z)$

where $q_{0}$ is a constant value taken by quantity $q$ within the region $D$. The substitution of (14) in (8), after differentiating the $\theta$-functions using (13), leads to the contour integral

$$
\begin{aligned}
\varepsilon_{i k} \frac{\partial}{\partial x_{i}} & \left(\psi-\omega \frac{r^{2}}{2}\right) \frac{\partial}{\partial x_{k}}\left(\Delta \psi-\frac{1}{R^{2}} \psi\right) \\
& =-4 q_{0}\left[\frac{\partial \theta}{\partial z} \frac{\partial}{\partial \bar{z}}\left(\psi-\omega \frac{r^{2}}{2}\right)\right] \\
& =q_{0} \oint d s \delta(\boldsymbol{x}-\hat{\boldsymbol{x}}) \frac{\partial}{\partial s}\left(\hat{\psi}-\omega \frac{|\hat{z}|^{2}}{2}\right)=0 .
\end{aligned}
$$

It follows from this substitution that the rotate-frame stream function must be constant on the vortex boundary:

$\hat{\psi}-\omega \frac{|\hat{z}|^{2}}{2}=$ const.

Here, $\hat{\psi}$ is defined by $\hat{\psi}=\psi_{\boldsymbol{x}=\hat{\boldsymbol{x}}}$.

Eq. (16) determines the boundary shape (10) if the stream function $\hat{\psi}$ is expressed in terms of the contour coordinates $\hat{\boldsymbol{x}}$. Taking into account that the Green's function $G\left(\boldsymbol{x}, \boldsymbol{x}^{\prime}\right)$ of operator $\Delta-R^{-2}$ is given by

$G\left(\boldsymbol{x}, \boldsymbol{x}^{\prime}\right)=-\frac{1}{2 \pi} K_{0}\left(\frac{\left|\boldsymbol{x}-\boldsymbol{x}^{\prime}\right|}{R}\right)$, 
and by solving (9), we obtain the stream-function $\psi$ in terms of the cyclostrophic potential vorticity $q$ :

$\psi(\boldsymbol{x})=-\frac{1}{2 \pi} \int d \boldsymbol{x}^{\prime} q\left(\boldsymbol{x}^{\prime}\right) K_{0}\left(\frac{\left|\boldsymbol{x}-\boldsymbol{x}^{\prime}\right|}{R}\right)$,

where $d \boldsymbol{x}=d x_{1} d x_{2}$ and $K_{n}(\xi)$ denotes a modified Bessel function of the $n$-th order.

To convert (17) into a contour integral, we make use of the equality

$$
\begin{aligned}
K_{0} & =R^{2} \Delta K_{0}+2 \pi R^{2} \delta\left(\boldsymbol{x}-\boldsymbol{x}^{\prime}\right) \\
& =4 R^{2} \frac{\partial^{2} K_{0}}{\partial \bar{z} \partial z}+2 \pi R^{2} \delta\left(\boldsymbol{x}-\boldsymbol{x}^{\prime}\right),
\end{aligned}
$$

which follows immediately from the definition of the Green's function. Assuming that $\boldsymbol{x} \rightarrow \hat{\boldsymbol{x}}$, existing outside the region $D$, and integrating (17) in parts with obvious transformations, we find

$$
\begin{aligned}
\hat{\psi}(s) & =-\frac{2 R^{2}}{\pi} \int d \boldsymbol{x}^{\prime} q\left(\boldsymbol{x}^{\prime}\right) \frac{\partial^{2}}{\partial \bar{z}^{\prime} \partial z^{\prime}} K_{0}\left(\frac{\left|\boldsymbol{x}-\boldsymbol{x}^{\prime}\right|}{R}\right) \\
& =\frac{2 q_{0} R^{2}}{\pi} \int d \boldsymbol{x}^{\prime} \frac{\partial \theta^{\prime}}{\partial \bar{z}^{\prime}} \frac{\partial}{\partial z^{\prime}} K_{0}\left(\frac{\left|\hat{\boldsymbol{x}}-\boldsymbol{x}^{\prime}\right|}{R}\right) \\
& =\frac{R q_{0}}{2 \pi} \int d s^{\prime} K_{1}\left(\frac{\left|\hat{z}^{\prime}-\hat{z}\right|}{R}\right) \frac{\hat{z}_{s}^{\prime}\left(\bar{z}^{\prime}-\bar{z}\right)}{\left|\hat{z}^{\prime}-\hat{z}\right|} .
\end{aligned}
$$

Now we introduce the new variable $\varphi(s)$ which is the slope angle that is formed when the unit vector is tangential to the contour at a point $s$ with the axis $x_{1}$. Then, according to (11), we have

$\partial_{s} \hat{z}=e^{i \varphi(s)}$.

For vortex structures with a weak enough contour curvature

$|\kappa|=\left|\partial_{s} \varphi\right| \ll 1 / R$,

It is possible to make the following approximations in the integral of (18)

$\left|\hat{z}^{\prime}-\hat{z}\right| \approx\left|s^{\prime}-s\right|, \quad \operatorname{Im} \hat{z}_{s}^{\prime}\left(\bar{z}^{\prime}-\bar{z}\right) \approx \frac{1}{2} \kappa(s)\left(s^{\prime}-s\right)^{2}$,

where an over-bar denotes a complex conjugation.

With the help of (21), the integral (18) can be reduced to the local relation

$\hat{\psi}(s)=\frac{q_{0} R^{3}}{4} \kappa(s)$,

which relates the stream function $\hat{\psi}$ and the contour curvature $\kappa$ in the point $s$.

\section{Equations for contour curvature}

To derive the equations which define the boundary shape in the $z$-plane, we make the transformation

$\hat{z}=e^{i \varphi}(\hat{x}+i \hat{y})$, where $\hat{x}$ and $\hat{y}$, as well as $\varphi$ are some functions of the contour arc length $s$. Then from (19), the following relationships hold:

$\frac{\partial \hat{x}}{\partial s}-\kappa \hat{y}=1, \quad \frac{\partial \hat{y}}{\partial s}+\kappa \hat{x}=0$.

To obtain a complete description of the contour, we substitute (22) and (23) in (16):

$\frac{q_{0} R^{3}}{2 \omega} \kappa-\left(\hat{x}^{2}+\hat{y}^{2}\right)=$ const.

For convenience, we non-dimensionalize the contour Eqs. (24) and (25) by taking as a length scale

$L=\frac{R}{2}\left(\frac{q_{0}}{\omega}\right)^{1 / 3}$.

In terms of the dimensionless variables, without changing the old symbol designations, we obtain the following equations:

$\frac{\partial \hat{x}}{\partial s}-\kappa \hat{y}=1, \quad \frac{\partial \hat{y}}{\partial s}+\kappa \hat{x}=0$,

$\kappa-\frac{1}{4}\left(\hat{x}^{2}+\hat{y}^{2}\right)=$ const.

From (27) we can readily obtain the expressions for the variables $\hat{x}$ and $\hat{y}$ in terms of curvature:

$\hat{x}=2 \frac{\partial \kappa}{\partial s}, \quad \hat{y}=2 c_{1}-\kappa^{2}$.

Then, the substitution (28) in (27) gives the equation for the curvature:

$\left(\frac{\partial \kappa}{\partial s}\right)^{2}=-\frac{1}{4} \kappa^{4}+c_{1} \kappa^{2}+\kappa+c_{2}$.

Here, $c_{1}$ and $c_{2}$ are two constants that parameterize the solutions of the problem.

Recall that the condition for application of Eq. (29) is determined by the inequality (20), which in dimensionless form is given by

$|\kappa| \ll \frac{L}{R}=\frac{1}{2}\left|\frac{q_{0}}{\omega}\right|^{1 / 3}$.

As this inequality shows, the condition of the weak contour curvature does not limit the physical applicability of the solutions, as it might appear at first sight. The reason is that the inequality (30) always holds for intense vortices which are characterized by large enough values of the ratio $q_{0} / \omega$.

According to the theory of elliptic functions (Abramovitz and Stegun, 1964; Bateman and Erdelui, 1955), Eq. (29) has two sets of the periodic solutions expressed in terms of elliptic functions

$\kappa=b+\frac{a-b}{1-\alpha \mathrm{F}(\lambda s \mid m)}$.

Here, $\mathrm{F}$ is one of the Jacobi elliptic functions, and sn or $\mathrm{dn}$, and $m$ are the parameters of these functions (a vertical line symbolizes the $m$-dependence). Note that depending on which Jacobi elliptic function is taken, the independent basic parameters $\alpha$ and $m$ will parameterize $a, b, \lambda$, and consequently, $c_{1}$ and $c_{2}$. 


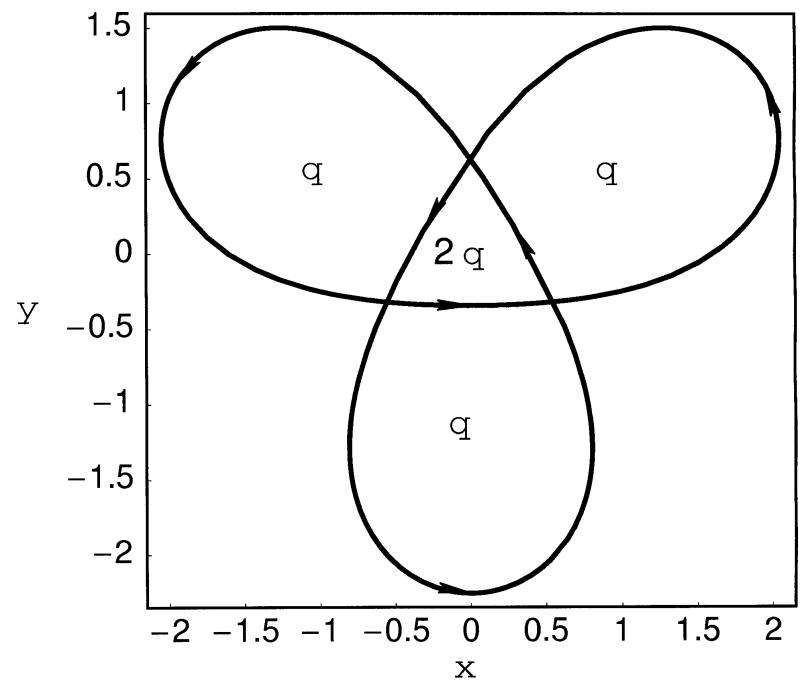

Fig. 1. The geometry of the three-petal vortex region of the piece wise constant vorticity with self-intersecting contour. The distribution of the cyclostrophic potential vorticity is $q_{0}$ in petals and $2 q_{0}$ in the core, so the jump in the potential vorticity is the invariant $q_{0}$ in tracing the contour.

\section{$6 \quad N$-petal vortex structures}

In the case $F=\operatorname{sn}(\lambda \mathrm{s} \mid \mathrm{m})$, the periodic solution (31) for the contour curvature takes the form

$\kappa=b+\frac{a-b}{1-\alpha \operatorname{sn}(\lambda s \mid m)}$,

where

$a=-2^{-1 / 3} \frac{\alpha\left(1+m-2 \alpha^{2}\right)}{\left[(1-m)^{2} \alpha\left(m-\alpha^{4}\right)\right]^{1 / 3}}$,

$b=2^{-1 / 3} \frac{\alpha^{2}+m\left(\alpha^{2}-2\right)}{\alpha\left[(1-m)^{2} \alpha\left(m-\alpha^{4}\right)\right]^{1 / 3}}$,

$\lambda=2^{-1 / 3} \frac{\sqrt{\left(\alpha^{2}-m\right)\left(1-\alpha^{2}\right)}}{\left[(1-m)^{2} \alpha\left(m-\alpha^{4}\right)\right]^{1 / 3}}$.

In turn, the parameters $c_{1}$ and $c_{2}$ are expressed in terms of $a$ and $b$ as

$c_{1}=\frac{b a}{2}-\frac{1}{a+b}, \quad c_{2}=-\frac{1}{4}\left(b+a+b^{2} a^{2}\right)$.

It should be emphasized that the conditions for contour continuity (smoothness) and the reality of the solutions for Eq. (30) impose the following restrictions on the parameters $\alpha$ and $m$ :

$0 \leq \alpha \leq 1, \quad m<\alpha^{2}$.

It follows from the formula (23) that in order to find the boundary shape, we must know the slope angle $\varphi(s)$ in addition to the variable $\kappa$. also the variable $\hat{x}$ and $\hat{y}$. This can be computed by integrating (32) along the contour line,

$\varphi(s)=\int^{s} d s^{\prime} \kappa\left(s^{\prime}\right)$

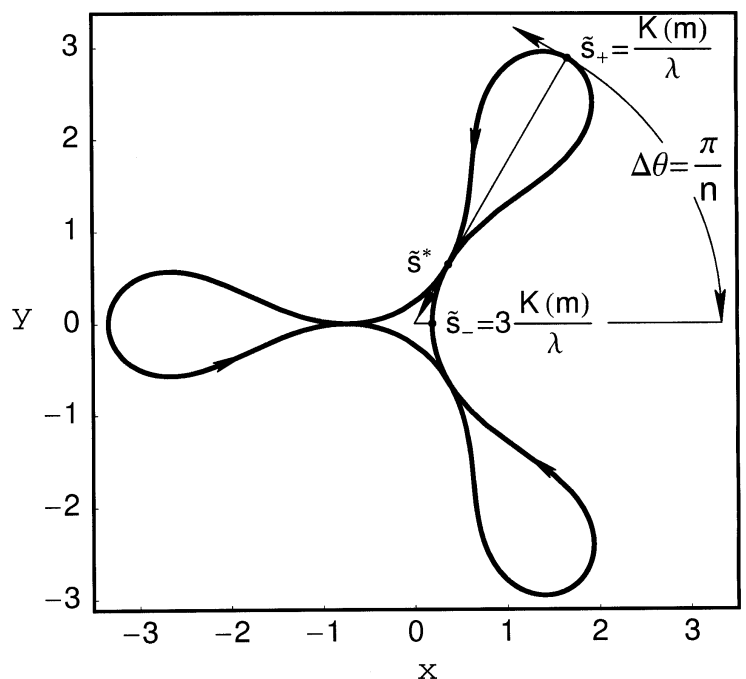

Fig. 2. Three-petal vortex structure. The point $s_{+}=K(m) / \lambda$ lies at the top of the petal and $s_{-}=3 K(\mathrm{~m}) / \lambda$ lies between the petals. $s^{*}$ is the self-contacting point of the contour.

$$
\begin{aligned}
= & b s+\frac{a-b}{\lambda} \Pi\left(\alpha^{2} ; \operatorname{am}(\lambda s \mid m) \mid m\right) \\
& -2 \operatorname{Im} \ln \left[\operatorname{cn}(\lambda s \mid m) \sqrt{\alpha^{2}-m}\right. \\
& \left.+i \operatorname{dn}(\lambda s \mid m) \sqrt{1-\alpha^{2}}\right] .
\end{aligned}
$$

Here, $\Pi(u ; \vartheta \mid m)$ is the incomplete elliptic integral of the third kind and the Jacobi amplitude am $(u \mid m)$ is the function defined by $\operatorname{am}(u \mid m)=\arcsin (\operatorname{sn}(u \mid m))$.

We shall restrict our study to the vortex structures with a finite area bounded by a closed contour without selfintersections. The precise equations of two-dimensional fluid dynamics allow for the existence of solutions with such a topology of a contour. Self-intersecting contours correspond to rather exotic vortex formations. To consider such contours some generalizations of the model assumptions should be made in the initial statement of the problem. Since the vortex region becomes multiply connected due to self-intersection, the corresponding piece wise constant vorticity distribution may be found. If the topology of the self-intersecting contour is known, the distribution of the cyclostrophic potential vorticity can be easily reproduced because the jump in the potential vorticity must remain invariant when going around the contour in one of the directions (see Fig. 1). In essence, the question of whether to include the self-intersecting solutions into the framework of our scheme is a question of whether or not the global character of the solutions is sensitive to a local violation of the approximation of a weak curvature. The answer can be found from comparing the numerical and analytical solutions.

Since the contour is closed and its curvature is a periodic function in the arc length $s$, the boundary shape of the vortex distributions under consideration has an $n$-petal structure. An example of such a structure is illustrated in Fig. 2. From this figure and the analysis of the solution (32), it is clear that the contour curvature of the $n$-petal structure has extrema at the 
points, since it is a periodic function with period $4 K(m) / \lambda$ :

$s_{-}=(4 j-1) \frac{K(m)}{\lambda}$,

$s_{+}=(4 j-3) \frac{K(m)}{\lambda}, \quad j=1,2, \ldots, n$,

where $K(m)$ is a complete elliptic integral of the first kind. At these points the contour curvature takes the extreme values:

$\kappa_{+}=b+\frac{a-b}{1-\alpha}=2^{-1 / 3} \frac{m(\alpha+2)-\alpha(1+2 \alpha)}{\left[(1-m)^{2} \alpha\left(m-\alpha^{4}\right)\right]^{1 / 3}}$,

$\kappa_{-}=b+\frac{a-b}{1+\alpha}=2^{-1 / 3} \frac{m(\alpha-2)-\alpha(1-2 \alpha)}{\left[(1-m)^{2} \alpha\left(m-\alpha^{4}\right)\right]^{1 / 3}}$.

The subscript notation $\mp$ means that $f_{\mp}=f\left(s_{\mp}\right)$. The relative position of the turning points $s_{-}$and $s_{+}$depends on the parameters $\alpha$ and $m$. In order to establish which of these parameters is at the tip of the petals and which is in the trough between the petals, it is necessary to calculate the distances of these points to the symmetry center. For this purpose we introduce the polar coordinates of point $s$ as $\rho$ and $\theta$ so that $\hat{z}(s)=\rho e^{i \theta}$.

According to the above results, the variables $\rho$ and $\theta$ are given by

$\rho^{2}=4\left(c_{1}^{2}+c_{2}+\kappa\right)$,

$\theta=\operatorname{arcctg}\left(\frac{\partial \kappa / \partial s}{c_{1}-\kappa^{2} / 2}\right)+\varphi$.

Expressing $c_{1}, c_{2}$ in terms of $\alpha$ and $m$ through (34), (33) and substituting these expressions together with (37) in (38), we find that

$\rho_{+}^{2}=2^{2 / 3} \frac{\left[m(1+2 \alpha)-\alpha^{3}(\alpha+2)\right]^{2}}{\alpha\left(m-\alpha^{4}\right)\left[(1-m)^{2} \alpha\left(m-\alpha^{4}\right)\right]^{1 / 3}}$,

$\rho_{-}^{2}=2^{2 / 3} \frac{\left[m(1-2 \alpha)-\alpha^{3}(\alpha-2)\right]^{2}}{\alpha\left(m-\alpha^{4}\right)\left[(1-m)^{2} \alpha\left(m-\alpha^{4}\right)\right]^{1 / 3}}$.

The relative position of the turning points depends on whether 1 is greater or less than the ratio

$\left(\frac{\rho_{-}}{\rho_{+}}\right)^{2}=1+\frac{8 \alpha\left(\alpha^{2}-m\right)\left(m-\alpha^{4}\right)}{\left.\left[m(1+2 \alpha)-\alpha^{3}(\alpha+2)\right]\right]^{2}}$.

It is easy to see that the inequality $\alpha^{4} \leq m \leq \alpha^{2}$ entails the inequality $\rho_{-} \geq \rho_{+}$, so in this interval of parameters the tops of the petals lie at the point $s_{-}$. In the event that $m \leq \alpha^{4}$ and consequently the reverse inequality $\rho_{-} \leq \rho_{+}$holds true, the tips of the petals lie at the point $s_{+}$.

It is obvious that not all solutions (32) in the region of permissible parameters

$m<\alpha^{2} \leq 1$

correspond to vortex structures with closed contours. The condition that a contour corresponding to a periodic solution (32) is closed can be formulated as

$\Delta \theta=\theta_{-}-\theta_{+}=\frac{\pi}{n}$.

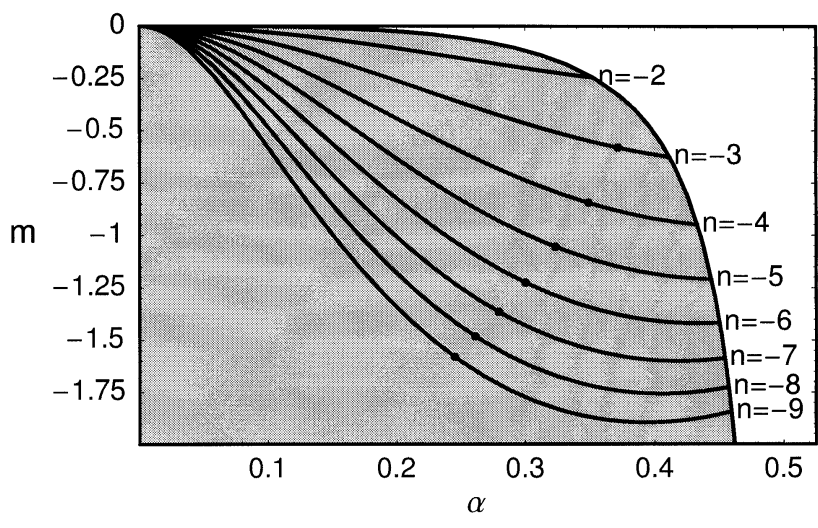

Fig. 3. The family of $n$-petal vortex regimes in the plane $(\alpha, m)$. The characteristic curves assign the dependence $m_{n}(\alpha)$ for $n=$ $-1,-2, \cdots,-9$. Limit points in which the corresponding vortex structure has the contour with a self-contact are marked as $\bullet$.

The requirement (43) has a simple geometrical meaning shown in Fig. 2. From this figure it is easy to see that $2 \Delta \theta$ is simply the angular distance between neighboring petals. To evaluate its value it suffices to note that the position vector and tangent are mutually orthogonal in turning points. Therefore,

$\theta_{ \pm}=\varphi_{ \pm}+\frac{\pi}{2} \Delta_{ \pm}$

where the signum function $\Delta_{ \pm}$is defined by the first term of (39). From the analysis,

$\Delta_{ \pm}=\operatorname{sign}\left[m(1 \pm 2 \alpha)-\alpha^{3}(\alpha \pm 2)\right]$.

The expression for $\varphi_{ \pm}$can be easily found from (36):

$\varphi_{ \pm}=(4 j-2 \mp 1)\left[b \frac{K(m)}{\lambda}+\frac{a-b}{\lambda} \Pi\left(\alpha^{2} \mid m\right)\right]-\pi$

where $\Pi(u \mid m)=\Pi\left(u ; \frac{\pi}{2} \mid m\right)$ is the complete elliptic integral of the third kind.

The above formulas allow us to rewrite (43) as

$b \frac{K(m)}{\lambda}+\frac{a-b}{\lambda} \Pi\left(\alpha^{2} \mid m\right)=\frac{\pi}{2}\left(\frac{1}{n}-\Delta\right)$,

where

$$
\begin{aligned}
\Delta= & \frac{1}{2}\left(\Delta_{-}-\Delta_{+}\right)= \\
= & \frac{1}{2}\left\{\operatorname{sign}\left[m(1-2 \alpha)-\alpha^{3}(\alpha-2)\right]\right. \\
& \left.-\operatorname{sign}\left[m(1+2 \alpha)-\alpha^{3}(\alpha+2)\right]\right\} .
\end{aligned}
$$

Equation (47) has solutions in the form of $n$-petal structures in region

$m \leq \alpha^{3} \frac{\alpha-2}{1-2 \alpha}$,

where $\Delta=0$ for $n \leq-2$. In Fig. 3 this region is marked by a shaded background. The solutions in this region are 


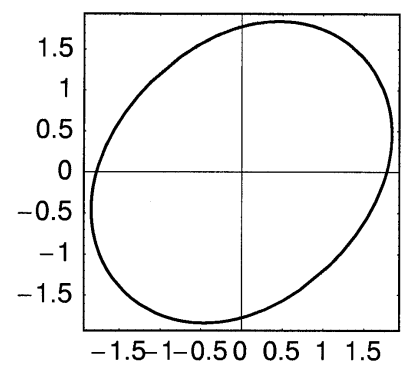

(a)

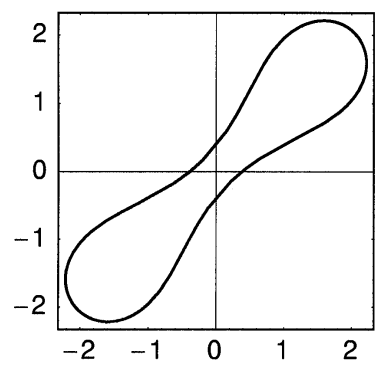

(c)

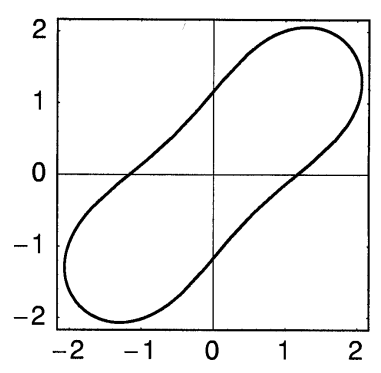

(b)

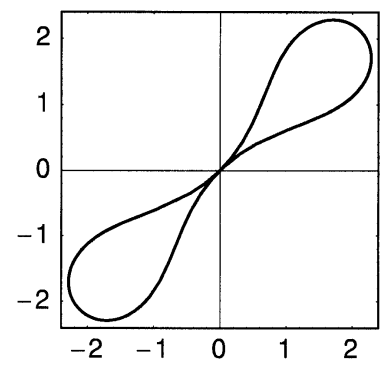

(d)
Fig. 4. Shapes of the boundaries for double petal vortex structures in the $x-y$ plane. (a) $\alpha=0.050$; (b) $\alpha=0.200$; (c) $\alpha=0.300$; (d) $\alpha=0.353$.

parameterized by characteristic curves which assign the dependence $m_{n}(\alpha)$ for every $n$. Hence an $n$-petal structure can be characterized by the one parameter $\alpha$. The vortex shapes for $n=2,3$, depending on $\alpha$, is shown in Figs. 4 and 5 . For every $n$-petal regime the characteristic curve has a limit point in which the corresponding vortex structure has a selfcontacting contour. To the left of the point there are solutions without self-intersections of contours and to the right, there are solutions with self-intersections.

The conditions for a self-contacting contour can be formulated on geometrical grounds which follow from Fig. 2. First of all, we note that in the tangency point $s^{*}$, the angles $\theta$ and $\varphi$ are related by the equality

$\theta\left(s^{*}\right)=\varphi\left(s^{*}\right)$.

Hence from (39), we immediately have $\kappa^{2}\left(s^{*}\right)=2 c_{1}$. One more condition is obtained if one takes into account that in tracing the contour from the point $s_{+}$to the tangency point $s^{*}$, the tangent vector is rotated through $\pi / 2$; hence $\varphi\left(s^{*}\right)-$ $\varphi_{+}=\pi / 2$. Making use of (28) and the relation

$\varphi_{+}=\frac{\pi}{2}\left(\frac{1}{n}-\Delta\right)-\pi$,

which follows from (46) and (47) at $j=1$, we obtain the conditions

$\varphi\left(s^{*}\right)=\frac{\pi}{2}\left(\frac{1}{n}-\Delta-1\right)$,

$\kappa^{2}\left(s^{*}\right)$

$=2^{-2 / 3} \frac{2 \alpha^{4}(1+m)+\alpha^{2}(1+m(m-10))+2 m(m+1)}{\left[(1-m)^{2} \alpha\left(m-\alpha^{4}\right)\right]^{2 / 3}}$

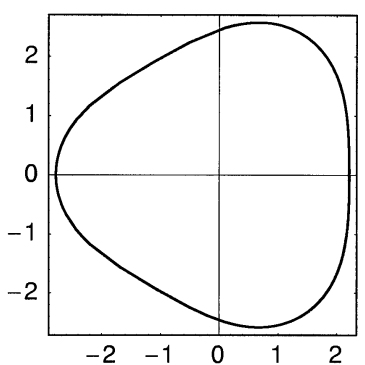

(a)

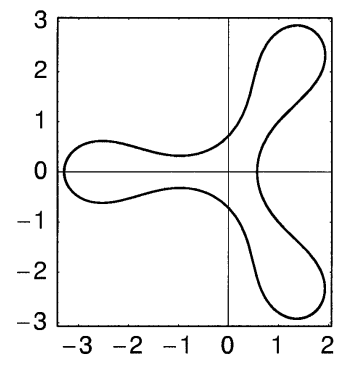

(c)

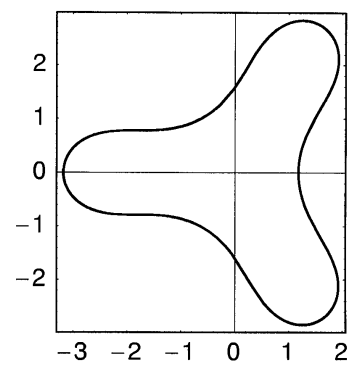

(b)

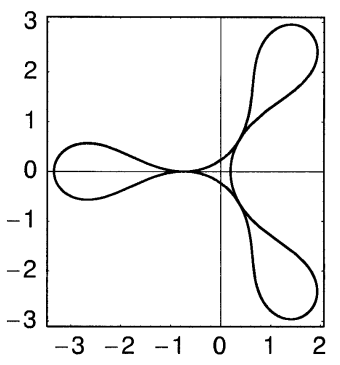

(d)
Fig. 5. Shapes of boundaries for the three-petal vortex structures in the $x-y$ plane. (a) $\alpha=0.050$; (b) $\alpha=0.200$; (c) $\alpha=0.300$; (d) $\alpha=0.371$.

These conditions, together with (47), fix all parameters of the limiting regime.

The case of dn-solutions may be considered analogously. However, in this case, only vortices with self-intersecting contours result.

\section{Temperature distribution}

The temperature distribution associated with these multi-petal vortex patches is determined by the quantity $\tau(\boldsymbol{x})$ which is connected to the stream function by the linear relation (7). This expression represents a deviation from the stationary background temperature distribution $\tau_{s}$. On the basis of the results obtained in Sect. 3, it is possible to establish the formula:

$$
\begin{aligned}
& \tau(\boldsymbol{x}) \\
& =-\tau_{0} \int d s\left(K_{1}\left(\frac{|\hat{z}-z|}{\varepsilon}\right)-\frac{\varepsilon}{|\hat{z}-z|}\right) \frac{\hat{z}_{s}(\bar{z}-\bar{z})}{|\hat{z}-z|},
\end{aligned}
$$

where $z=(x+i y) / L$ and $\tau_{0}=q_{0} \gamma R L / 2 \pi \omega, \varepsilon=R / L$.

The temperature distribution $\tau(\boldsymbol{x}) / \tau_{0}$ associated with the presence of this vortex and calculated in accordance with (50) is shown in Fig. 6. The radial temperature profiles corresponding to this vortex are presented in Fig. 7. As the cyclostrophic vortex rotates about the pole, its petals will be responsible for the temporal variations in temperature which may be detected by a stationary observer.

Since $L>0$ exists by definition, the cyclostrophic vortex has the same sense of rotation as the sign of its potential vorticity: $\operatorname{sign}(\omega)=\operatorname{sign}\left(q_{0}\right)$. Note also that as $\operatorname{sign}\left(\tau_{0}\right)>0$ 


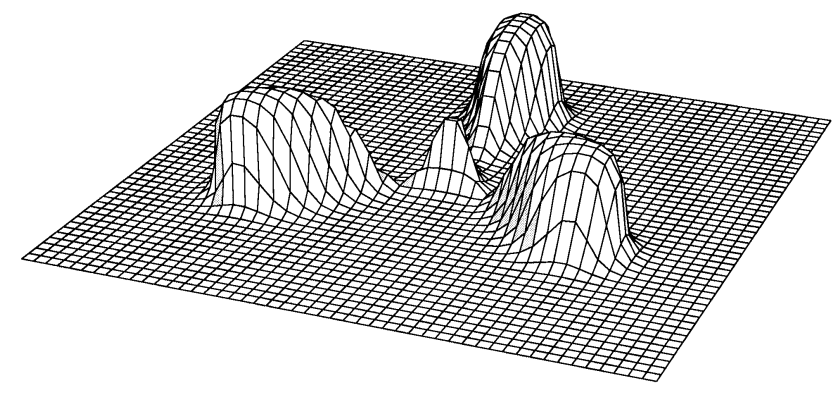

Fig. 6. Surface plot of the temperature field for the limiting threepetal cyclostrophic vortex.

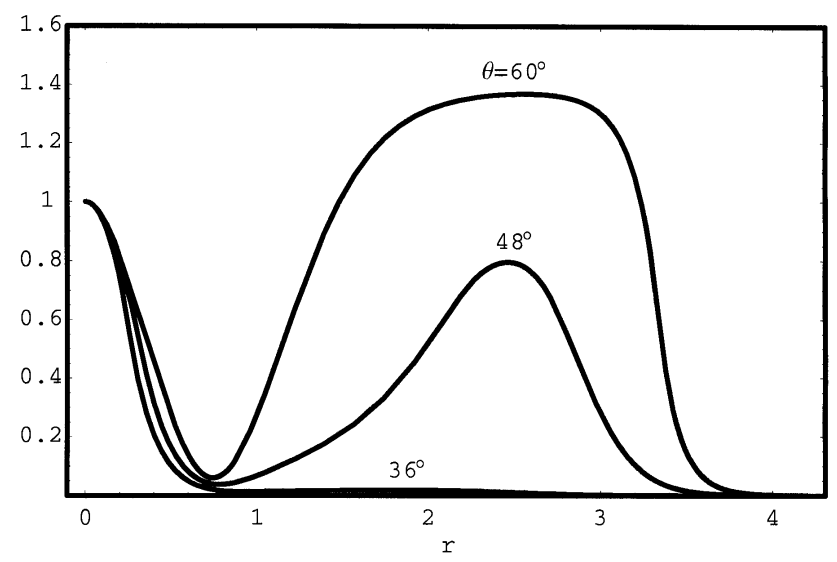

Fig. 7. The radial temperature profile for the limiting three-petal cyclostrophic vortex given in Fig. 6. The profiles correspond to the directions $\theta=60,36,48$.

both for $\omega>0$ and for $\omega<0$, the variations in temperature are always positive.

Let us compare the magnitudes of the parameters $\tau_{0}, \omega, \varepsilon$ with the observed data. The corresponding relations can be rewritten in the following form

$$
\begin{aligned}
& \frac{q_{0}}{\omega}=8\left(\frac{L}{R}\right)^{3}, \quad R=\frac{|\omega|}{\Omega \sqrt{\gamma \beta}}, \\
& \gamma=\frac{8 \Delta T}{\pi^{2} R_{0}^{2}}, \quad \beta=\frac{1}{T} .
\end{aligned}
$$

Here, $\Delta T$ is the pole-equator difference of temperatures, $T$ is the average temperature of the atmosphere, $R_{0}$ is the planet radius. The first three relations (7) follow from our theory and the last one assumes that the atmosphere is an ideal gas.

The ratio $\omega / \Omega$ is given in this case by the expression

$$
\left(\frac{\omega}{\Omega}\right)^{2}=\frac{256}{\pi^{5}} \frac{\Delta T}{\tau_{0}}\left(\frac{L}{R_{0}}\right)^{4} \frac{\Delta T}{T} .
$$

On the other hand, we find that $R=(\pi / 2 \sqrt{2}) R_{0}(|\omega| / \Omega)$ $\sqrt{T / \Delta T}$.

Let $L \sim 400 \mathrm{~km}, \quad R_{0} \sim 6 \cdot 10^{3} \mathrm{~km}, T \sim 600 \mathrm{~K}$, $\Delta T \sim 100 \mathrm{~K}, \tau_{0} \sim 10 \mathrm{~K}$ (Venusian "polar hot cap").
Table 1. Parameter values in limiting regimes

\begin{tabular}{ccccccc}
\hline$n$ & $\alpha$ & $m$ & $\kappa_{-}$ & $\kappa_{+}$ & $\rho_{-}$ & $\rho_{+}$ \\
\hline-2 & 0.353 & -0.246 & -0.457 & 1.791 & 0. & 2.120 \\
-3 & 0.371 & -0.581 & -0.820 & 1.953 & 0.194 & 3.336 \\
-4 & 0.349 & -0.844 & -1.016 & 2.031 & 0.424 & 3.517 \\
-5 & 0.324 & -1.055 & -1.158 & 2.089 & 0.636 & 3.660 \\
-6 & 0.300 & -1.225 & -1.273 & 2.139 & 0.830 & 3.786 \\
-10 & 0.231 & -1.666 & -1.600 & 2.299 & 1.497 & 4.224 \\
\hline
\end{tabular}

The substitution of these values into (52) yields an estimate $\omega / \Omega \sim 4,4 \cdot 10^{-3}$. In this case, $R \sim 50-100 \mathrm{~km} \ll L$.

According to Table 1 for the limiting cyclostrophic vortex with $n=2$, we have $\rho_{+}=2,12$, for the limiting cyclostrophic vortex with $n=3$, we have $\rho_{+}=3.37$, etc. Thus, each petal measures $\rho_{+} L \sim 1000 \div 1300 \mathrm{~km}$ radially in length.

Let us note that the multi-petal vortex configurations are sometimes found on either side of the Earth's poles at midwinter (see, e.g. the contours given by Palmen and Newton, 1969; Gill, 1982).

Acknowledgements. We would like to acknowledge the useful comments of A. Dyment. This work was partially supported by the Russian Fundamental Research Foundation under grant No. 00-0564019.

\section{References}

Abramovitz, M. and Stegun, I. A., Handbook of Mathematical Functions, U. S. Govt. Printing Office, Washington, DC, 1964.

Bateman, H. and Erdelyi, A., Higher Transcendental Function, McGraw-Hill Book Company, New York, Toronto, London, 1955.

Dritschel, D. G., Contour surgery: a contour dynamics method for long-time behaviour of two-dimensional, inviscid, rotational flow, J. Comput. Phys., 77, 240-266, 1988a; Contour dynamics/surgery on the sphere, J. Comput. Phys., 79, 477-483, 1988b.

Dritschel, D. G. and McIntyre, M. E., Does contour dynamics go singular? Phys. Fluids A, 2 (5), 748-753, 1990.

Dritschel, D. G. and Legras, B., Modeling oceanic and atmospheric vortices, Phys. Today, 46 (3), 44, 1993.

Frisch, U., Turbulence, Cambridge University Press, 1995.

Gill, A. E., Atmosphere-Ocean Dynamics, Academic Press, 1982.

Goncharov, V. P. and Pavlov, V. I., Vortex structures generated by a coastal currant in harbour-like basins at large Reynolds numbers, J. Fluid Mech., 351, 201-221, 1997.

Goncharov, V. P. and Pavlov, V. I., Two-dimensional vortex motions of fluid at large Reynolds numbers, Phys. Fluids, 10 (9), 2384 2395, 1998.

Goncharov, V. P. and Pavlov, V. I., Large-scale vortex structures in shear flows, Eur. J. Mech. B/Fluids, 19, 831-854, 2000.

Goncharov, V. P. and Pavlov, V. I., Some estimates on the space scales of vortex paires emitted from river mouths, Nonlin. Proc. in Geophysics, 8, 1-7, 2001.

Greenspan H. P., The Theory of Rotating Fluids, Cambridge University Press, 1968. 
Gryanik, V. M., The vertical nature of hot spot in the polar region of the atmosphere of Venus, Sov. Phys. Dokl., 35 (7), 592-595, 1990.

Holton, J. R., An Introduction to Dynamic Meteorology, 3rd ed., Academic Press, 1992.

Hunten, D. M., Colin, L., Donahue, T. M., and Moroz, V. I., Venus, The University of Arizona Press, 1983.

Ingersoll, A. P., Atmospheric dynamics of the other planets, Science, 248, 308, 1990.

Kamenkovich, V. M., Fundamentals of Ocean Dynamics, Elsevier, 1977.

Landau, L. D. and Lifshitz, E. M., Fluid Mechanics, 2nd ed., rev., Pergamon Press, Oxford, New York, 1987.

Lavrent'ev, M. A. and Shabat, B. V., Hydrodynamic Problems and their Mathematical Models, Nauka, Moscow, 1973.

Madelung, E., Die mathematischen Hilfsmittel des Physikers, Springer-Verlag, Berlin, Gottingen, Heidelberg, 1957.

Marcus, P. S., Numerical simulations of Jupiter's Great Red Spot, Nature, 331, 693, 1988.

Maslov V. P., Operational Methods, Nauka, Moskow, 1973.

Milne-Thomson, L. M., Theoretical Hydrodynamics, 5th edition, MacMillan, London, 1968.

Palmen, E. and Newton, C. W., Atmospheric Circulation Systems, Academic, New York, 1969.

Pavlov, V. I., Buisine, D., and Goncharov, V. P., Formation of vortex clusters on a sphere, Nonlin. Proc. in Geophysics, 8, 9-19, 2001.

Pedlosky, J., Geophysical Fluid Dynamics, Springer-Verlag, New York, 1987.

Robinson, A. R., Editor, Eddies in Marine Scences, Springer, Berlin, 1983.

Saffman, P. G., Vortex Dynamics, Cambridge University Press, 1992.

Schubert, G. General circulation and the dynamical state of the Venus atmosphere, in Venus, Eds. Hunten, D. M., Colin, L., Donahue, T. M., and Moroz, V. I., The University of Arizona Press, 1983.

Seiff, A., Thermal structure of the atmosphere of Venus, in Venus, Eds. Hunten, D. M., Colin, L., Donahue, T. M., and Moroz, V. I., The University of Arizona Press, p. 237, 1983.

Sommeria, J., Meyers, S. P., and Swinney, H. L. Laboratory simulation of Jupiter's Great Red Spot, Nature, 331, 689, 1988.

Taylor, F. W., et al., Structure and meteorology of the middle atmosphere of Venus: Infrarared remote sensing from the Pioneer orbiter, J. Geophys. Res., 85, 7963-8006, 1980.

Turner, J. S., Buoyancy effects in fluids, Cambridge University Press, 1979.

Zabusky, N. J., Huges, M. H., and Roberts, K. V., Contour dynamics for the Euler equations in two-dimensions, J. Comput. Phys., 30, 96-106, 1979. 